\title{
EDITORIAL
}

\section{Chemokines: the past, the present and the future}

\author{
Peng Tang ${ }^{1,2}$ and Ji Ming Wang ${ }^{2}$
}

Cellular and Molecular Immunology (2018) 15, 295-298; doi:10.1038/cmi.2018.9; published online 26 March 2018

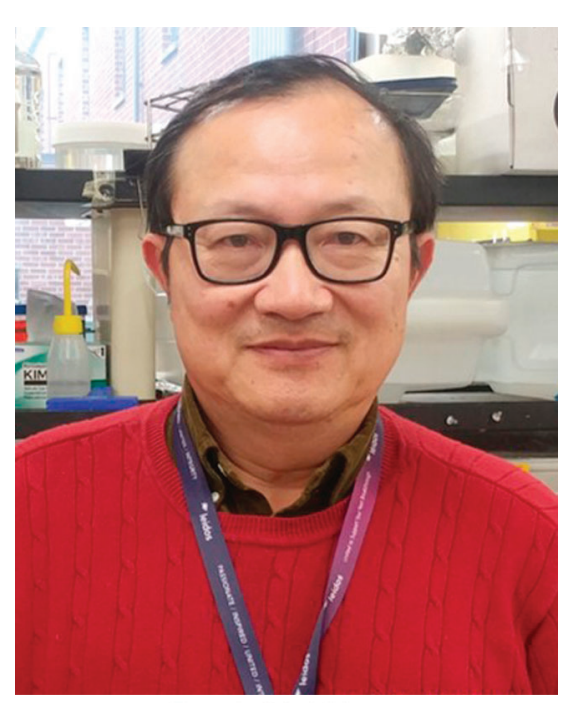

Dr. J. M. Wang

We are very excited to publish our article in the "Chemokine Special Issue" of Cellular and Molecular Immunology, which has received increasing attention in the field of immunology. A natural question emerging regarding this issue is, why chemokines? The answer may of course not be simple, but if we consider only the essential, a shortened answer

\footnotetext{
${ }^{1}$ Department of Breast Surgery, Southwest Hospital, Army Medical University, Chongqing 400038, China and ${ }^{2}$ Cancer and Inflammation Program, Center for Cancer Research, National Cancer Institute at Frederick, Frederick 21702, MD, USA

Correspondence: Dr JM Wang, Cancer and Inflammation Program, Center for Cancer Research, National cancer institute at Frederick, MD 21702, USA.

E-mail: wangji@mail.nih.gov

Received: 27 December 2017; Accepted: 2 January 2018
}

should be because chemokines are among the first mobilizers of host responses in homeostasis and diseases associated with inflammation (Figure 1).

The inflammatory response is a defensive mechanism that has evolved in higher organisms to localize and eliminate injurious agents and damaged tissue components. The response consists of changes in blood flow, an increase in the permeability of blood vessels, and the extravasation of fluid, proteins and leukocytes from the circulation to the inflamed site. Acute inflammation lasts only a few days, followed by resolution or healing of the affected tissue, while a prolonged response, referred to as chronic inflammation, is a culprit underlying many pathological conditions, including persistent pathogen infection, auto-immune diseases, cardiovascular diseases, diabetes, obesity, neurodegenerative diseases and cancer, which is often defined as a wound that does not heal. ${ }^{1,2}$

There are four cardinal signs of inflammation: redness (rubor), heat (calor), swelling (tumor) and pain (dolor), as described in the 1st century $\mathrm{AD}$ by the Roman medical historian Aulus Cornelius Celsus. A fifth sign of inflammation is the loss of function of the affected body area due to pain and distortion, as described by the German pathologist Rudolf Virchow in the 19th century.

A hallmark of inflammation is the accumulation of leukocytes at the site of insult. Although the old doctrine speculates that increased permeability of blood vessels allows for the exudation of leukocytes from the circulation to surrounding tissues, it is now well established that leukocyte extravasation in inflammatory responses is mediated by bacterial and host-derived chemoattractants, including a horde of small polypeptides named chemokines, a condensed term from chemotactic cytokines, which, by activating seven transmembrane G-protein coupled receptors (GPCRs) on the cell surface, guide a directional (i.e., chemotaxis), but not random (chemokinesis), migration of the cells.

To better understand the pathophysiological significance of chemokines, this special issue includes five review articles $^{3-7}$ that provide a glimpse of their classification, structure-function relationship and involvement in diseases. Due to space limitations, the content included in this special issue may reflect only the tip of an iceberg in the deep ocean. ${ }^{8-10}$ However, the enthusiasm this issue hopes to elicit in readers of the journal will further encourage greatly expanded studies with more profound depth.

Among modern biomedical sciences, chemokine research has a rather short but rich history. The first biologically active chemokines were discovered in the late 1980s and early 1990s, based on the presence of leukocyte chemoattractant activity present in the culture supernatants of human mononuclear leukocytes stimulated by proinflammatory agents such as bacterial endotoxin and cytokines, ${ }^{3}$ which demonstrated potent 


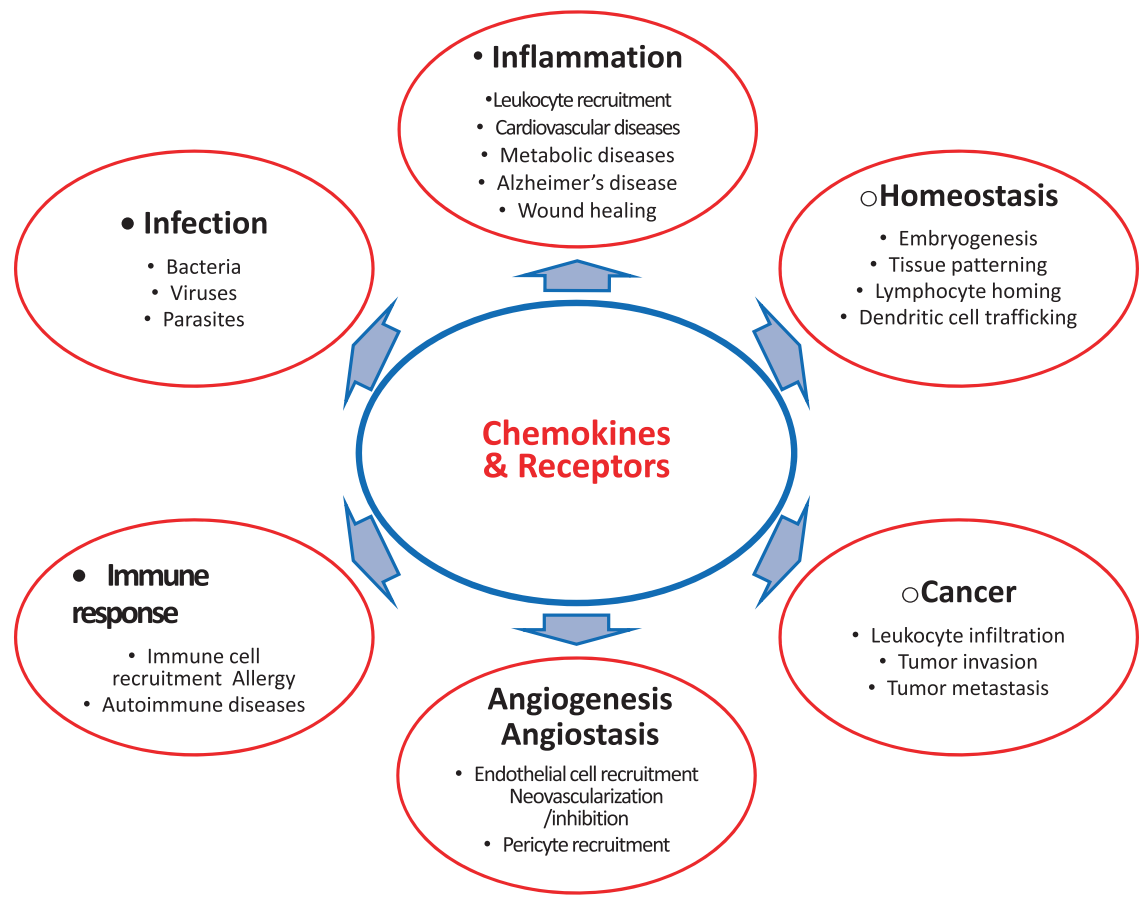

Figure 1 The role of chemokines and their GPCRs in homeostasis and disease. Chemokines and their GPCRs have been recognized as the key mediators of essential pathophysiological processes, including development, leukocyte trafficking and a plethora of diseases associated with inflammatory and immune responses. Chemokines and their GPCRs thus constitute plausible molecular targets for translational studies. GPCR, G-protein coupled receptor.

chemotactic activity for monocytic leukocytes. Yoshimura, one of the pioneers in the discovery of chemokines, detailed the isolation and characterization of monocyte chemotactic protein 1 (MCP-1), later termed CCL2. ${ }^{4}$ CCL2 is one of the most potent chemokines, preferentially inducing the accumulation and activation of monocytes/macrophages at sites of infection, inflammation and cancer. This discovery was followed by the identification in similar cell supernatants of another chemokine, IL-8, which is now termed CXCL8, ${ }^{3}$ with preferential chemotactic activity for neutrophils to induce rapid cell accumulation at sites of acute inflammatory responses. CCL2 and CXCL8 represent the chemokines obtained through classical biochemical purification procedures, which is rather labor intensive and time consuming, and has unpredictable results. However, low stringency hybridization technology using degenerative oligonucleotide probes, which was developed in early 1990s, enabled the identification of cDNA clones that encode, based on deduced protein sequences, novel putative chemokines and receptors. Recombinant proteins were then produced to test their binding and activating capacity in selected cell populations or cell lines transfected to express putative receptors, with the tentative name of "orphan receptors". Dozens of new chemokines have been identified in this manner, with matching target cells and receptors.

Ever increasing numbers of chemokines and GPCRs have prompted adoption of a new nomenclature. Chemokines are generally categorized as homeostatic and inflammatory chemokines or receptors based on their major in vivo functionalities. ${ }^{3,11}$ However, some chemokines and GPCRs display both homeostatic and inflammatory activities. One will also notice that there are often overlapping activities of chemokines or GPCRs, a feature of "promiscuity", that is, one chemokine may bind and activate more than one GPCR, and by analogy, one GPCR may recognize more than one singlechemokine ligands. This phenomenon is often interpreted as the ability of the chemokine system to compensate among one another during complex host responses in given circumstances, initially triggering speculation of redundancy in either chemokines or GPCRs. However, careful investigation, especially with genetically engineered mice overexpressing or being depleted of a ligand or GPCR, has revealed that each chemokine or receptor is endowed with unique functionality under pathophysiological conditions.

Currently, there is more intense interest in chemokines and receptors in vivo, with the aim of translation into drug targets. It has been established that in any disease with complex pattern of cell composition, such as in injured tissues, lymphatic organs and cancer, one or more chemokines and receptors are almost certainly involved. However, they may be either beneficial or detrimental to the host, based on the temporal and spatial context. For example, although CCL2 was originally identified as a monocyte chemoattractant during host responses to inflammatory stimulation, 
its involvement in host interaction with cancer may yield dual, or opposing, consequences. ${ }^{4}$ CCL2 contributes substantially to the recruitment of blood monocytes into tumors, where monocytes exhibit both tumoricidal and tumor-promoting activities. Since in vitro-cultured tumor cells often produce copious quantities of CCL2, tumor cells were once considered a main source. However, a variety of non-tumor cells in the tumor stroma also produce CCL2 in response to various stimulants. Therefore, the production of this chemokine in tumors becomes a consequence of complex interactions between tumor cells and non-tumor cells. Although CCL2 produced in tumors was originally considered a host anticancer defense, it is now well understood that this chemokine regulates a vicious cycle between tumor cells and immune-suppressive (M2) macrophages that promote tumor progression. Interestingly, certain tumor cells similar to monocytes express a GPCR (CCR2) for CCL2, which either mediates organ-specific dissemination of tumor cells ${ }^{3,11}$ or activates growth signals in tumor cells ${ }^{12}$ to facilitate epithelialmesenchymal transition, an indication of a more highly malignant and motile tumor cell phenotype. Therefore, efforts to disrupt the vicious cycle of the CCL2/ CCR2 axis in tumors have shown effects on enhancing anti-tumor host immunity and on reducing tumor progression.

Another prominent feature of chemokines in homeostasis and diseases is their ability to regulate the trafficking of dendritic cells (DCs), as described by Sozzani et al. ${ }^{5}$ DCs are professional antigen-presenting cells that are responsible for the activation of specific $\mathrm{T}$ and $\mathrm{B}$ lymphocyte-mediated immunity. The biological activities of DCs are closely related to the precise tissue localization of the cells during different steps of pathophysiological conditions. Immature DCs reside in peripheral tissues and are specialized for antigen capture. Mature DCs must migrate to secondary lymphoid organs to present antigens to naive $\mathrm{T}$ (B) cells. The fine-tuned localization of DCs is controlled by chemotactic and non-chemotactic signals that include pathogen-associated molecular patterns, damage-associated molecular patterns, complement, lipids and, more prominently, chemokines. These signals act both individually and, in most cases, in concert to establish multiple levels of regulatory mechanisms, such as synergy, relay and interactions with selected atypical chemokine GPCRs. ${ }^{3,5}$ This article thus provides an up-to-date overview of a sophisticated scenario that controls DC trafficking and activation in immune responses and anticancer host defense.

It has been realized that chemokines often cooperate with other pathogen or host factors to contribute to the progression and resolution of many diseases. The article by Van Damme et al..$^{5}$ reviews the involvement of chemokines in two human chronic respiratory diseases, namely, primary ciliary dyskinesia (PCD) and cystic fibrosis (CF), as examples of such cooperation. Airway infections recur in patients with $\mathrm{PCD}$ and $\mathrm{CF}$, which are characterized by persistent bacterial colonization and uncontrollable inflammation. Neutrophil accumulation in the airway of both diseases is associated with high levels of CXCL8, the most potent neutrophil-specific chemokine. However, despite their presence in high numbers in the airway, neutrophils fail to eliminate pathogens and resolve the infection in PCD and CF. Instead, activated neutrophils cause lung damage and promote a vicious cycle of disease progression. While the inflammatory process in CF airways is better characterized, the evolution of lung pathology in PCD is far from clear. Therefore, knowledge gleaned from $\mathrm{CF}$ is helpful for further investigations of PCD lungs. This should prompt the modification of current therapeutic regimens for both $\mathrm{CF}$ and PCD by specifically targeting CXCL8 and coexisting cytokines to reduce neutrophil infiltration into the airway, thereby reducing tissue damage.

Many chemokines are present in vivo in post-translationally modified forms that generate either receptor agonists with altered strength or antagonists to regulate pathophysiological functions. For example, the CXC chemokine CXCL12, originally termed stromal cell-derived factor (SDF1), displays an immense array of activities in embryogenesis, hematopoiesis, angiogenesis, autoimmunity ${ }^{13,14}$ and cancer progression based on its ability to induce the migration of hematopoietic progenitor cells, endothelial cells, most leukocytes and cancer cells by interacting mainly with the receptor CXCR4., ${ }^{3,7}$ CXCL12 has the unique property of existing in six splice variants in humans, each possessing a specific tissue distribution pattern and biological function. The article by Proost et al. ${ }^{7}$ introduces the mechanistic basis for controlled splice variant transcription and mRNA stability that determine the expression profile of CXCL12. CXCL12 binds to CXCR4 as well as, to a lesser extent, an atypical chemokine receptor $3 .^{3,15}$ Its binding to glycosaminoglycans in tissues and vascular endothelium allows for more effective presentation to passing leukocytes and in detrimental cases, to tumor cells, in the circulation. Proteolytic removal of NH2or $\mathrm{COOH}$-terminal amino acids, citrullination by peptidyl arginine deiminases of arginine residues, or nitration of tyrosine residues, all reduce CXCL12 activity. This illustrates the complexity of posttranslational modification elements that tune the activity of a given chemokine, such as CXCL12, to maintain an equilibrium in host responses.

More than three decades of research has unveiled chemokines and their GPCRs as central players in a sphere that rotates inside a biological galaxy of homeostasis and diseases (Figure 1) to determine the fate of host responses. Considerable progress has been achieved in our understanding of the biology of chemokines and their GPCRs in the initiation, progression and resolution of diseases. However, questions remain concerning their roles in the more precise context of the micro-anatomical environment in which the diseases evolve. In addition, increasingly more novel chemokines and receptors are emerging to enlarge the depot of these biological response modifiers. Thus, one may anticipate that the chemoattraction of a larger army of investigators with diverse interests and expertise in both basic research and clinics covering extensive aspects of inflammation, immunity, 
infection and cancer should enormously benefit human health.

\section{CONFLICT OF INTEREST}

The authors declare no conflict of interest.

\section{ACKNOWLEDGEMENTS}

We thank Drs Joost J Oppenheim and Keqiang Chen for reviewing the manuscript and Ms Laraine Main for secretarial assistance. This project has been funded in part by federal funds from the National Cancer Institute, National Institutes of Health, under Contract No.

HHSN261200800001E. PT is also funded in part by the China State Scholarship Fund (No. 201603170050) and the National Natural Science Foundation of China (No. 81302315).

1 Mantovani A. Cancer: Inflaming metastasis. Nature 2009; 457: 36-37.
2 Balkwill F, Charles KA, Mantovani A. Smoldering and polarized inflammation in the initiation and promotion of malignant disease. Cancer Cell 2005; 7: 211-217.

3 Chen K, Bao Z, Tang P, Gong W, Yoshimura $\mathrm{T}$, Wang JM. Chemokines in homeostasis and diseases. Cell Mol Immunol 2018; 15: 324-334.

4 Yoshimura T. The chemokine MCP-1 (CCL2) in host interaction with cancer: a foe or ally? Cell Mol Immunol 2018; 15: 335-345.

5 Tiberio L, Prete AD, Schioppa T, Sozio F, Bosisio D, Sozzani S. Chemokines and chemotactic signals in dendritic cell migration. Cell Mol Immunol 2018; 15: 346-353.

6 Cockx M, Gouwy M, Van Damme J, Struyf S. Chemoattractants and cytokines in primary ciliary dyskinesia and cystic fibrosis: key players in chronic respiratory diseases. Cell Mol Immunol 2018; 15: 312-323.

7 Janssens R, Struyf S, Proost P. The unique structural and functional features of CXCL12. Cell Mol Immunol 2018; 15: 299-311.

8 Nagarsheth N, Wicha MS, Zou W. Chemokines in the cancer microenvironment and their relevance in cancer immunotherapy. Nat Rev Immunol 2017; 17: 559-572.

9 Griffith JW, Sokol CL. Luster AD. Chemokines and chemokine receptors: positioning cells for host defense and immunity. Annu Rev Immunol 2014; 32: 659-702.

10 Schulz 0, Hammerschmidt SI, Moschovakis GL, Forster R. Chemokines and chemokine receptors in lymphoid tissue dynamics. Annual Rev Immunol 2016; 34: 203-242.

11 Zlotnik A, Burkhardt AM, Homey B. Homeostatic chemokine receptors and organspecific metastasis. Nat Rev Immunol 2011; 11: 597-606.

12 Li S, Lu J, Chen Y, Xiong N, Li L, Zhang J et al. MCP-1-induced ERK/GSK-3 $\beta / S n a i l$ signaling facilitates the epithelial-mesenchymal transition and promotes the migration of MCF-7 human breast carcinoma cells. Cell Mol Immunol 2017; 14: 621-630.

13 Zhao LD, Liang D, Wu XN, Li Y, Niu JW, Zhou $\mathrm{C}$ et al. Contribution and underlying mechanisms of CXCR4 overexpression in patients with systemic lupus erythematosus. Cell Mol Immunol 2017; 14: 842-849.

14 Godessart N, Kunkel SL. Chemokines in autoimmune disease. Curr Opin Immunol 2001; 13: 670-675.

15 Bachelerie F, Graham GJ, Locati M, Mantovani A, Murphy PM, Nibbs R et al. New nomenclature for atypical chemokine receptors. Nat Immunol 2014; 15: 207-208. 